\title{
Radiative flow and heat transfer of a fluid along an expandable-stretching horizontal cylinder
}

\author{
Tarek G. Emam ${ }^{1,2}$ (D)
}

Correspondence: tarek.emam@sci.
asu.edu.eg
${ }^{1}$ Department of Mathematics,
Faculty of Science and Arts -
Khulais, University of Jeddah,
Jeddah, Kingdom of Saudi Arabia
${ }^{2}$ Department of Mathematics,
Faculty of Science, Ain Shams
University, Cairo, Egypt

Correspondence: tarek.emam@sci. asu.edu.eg

Faculty of Science and Arts Khulais, University of Jeddah 2Department of Mathematics, Faculty of Science, Ain Shams

\begin{abstract}
The effect of thermal radiation and suction/injection on heat transfer characteristics of an unsteady expandable-stretched horizontal cylinder has been investigated. Similarity equations are obtained through the application of similarity transformation techniques. The governing boundary layer equations are reduced to a system of ordinary differential equations. Mathematica has been used to solve such system after obtaining the missed initial conditions. The fluid velocity and temperature, within the boundary layer, are plotted and discussed in details for various values of the different parameters such as the thermal radiation parameter, suction/injection parameter, and unsteadiness parameter. Comparison of obtained numerical results is made with previously published results in some special cases and found to be in a good agreement. The obtained results show that the fluid velocity and temperature are affected by the variation of the parameters included in the study such as the radiation parameter, the unsteadiness parameter, and the suction/injection parameter.
\end{abstract}

Keywords: Heat transfer, Stretching cylinder, Thermal radiation, Suction and injection PACS: $44.40 .+a 44.90 .+c 44.05 .+e 47.15 .-x$

\section{Introduction}

Several applications in engineering and industrial processes arise from the study of the flow of either Newtonian fluid or non-Newtonian fluid. Such fields have been interesting for many authors for the last few decades. The fields of plastic and metallurgy industries, the drawing of wires, and glass fiber production are good examples for the applications of the problem of the flow over a stretching/shrinking cylinder.

The problem of the flow inside a tube that has a time-dependent diameter was first presented by Uchida and Aoki [1] and Shalak and Wang [2]. Wang [3] have studied the steady flow of incompressible viscous flow outside a hollow stretching cylinder. Elbashbeshy et al. [4] have investigated the effect of magnetic field on flow and heat transfer over a stretching horizontal cylinder in the presence of a heat source/sink with suction/injection. Hayat et al. [5] have examined the effects of variable thermal conductivity in mixed convection flow of viscoelastic nanofluid due to a stretching cylinder with a heat source/sink. Ishak et al. [6] have studied the MHD flow and heat

(c) The Author(s). 2019 Open Access This article is distributed under the terms of the Creative Commons Attribution 4.0 International License (http://creativecommons.org/licenses/by/4.0/), which permits unrestricted use, distribution, and reproduction in any medium, provided you give appropriate credit to the original author(s) and the source, provide a link to the Creative Commons license, and indicate if changes were made. 
transfer outside a stretching cylinder. They have got numerical solutions to the problem using the Keller-box method.

The thermal radiation effect is considerable when the difference between the surface temperature and the ambient temperature is big. Mabood et al. [7] have presented a theoretical investigation of flow and heat transfer of a Casson fluid from a horizontal circular cylinder in a non-Darcy porous medium under the action of slips and thermal radiation parameters.

Zaimi et al. [8] have studied the unsteady flow due to a contracting cylinder in a nanofluid using Buongiorno's model. Elbashbeshy et al. [9] have studied the effects of thermal radiation, heat generation, and suction/injection on the mechanical properties of the unsteady continuous moving cylinder in a nanofluid.

Fang et al. [10] have recently studied the problem of unsteady viscous flow over an expanding stretching cylinder which gives exact similarity solution to the Navier-Stokes equations. They found that the reversal flow fluid is strongly affected by the Reynolds number and the unsteadiness parameter. The numerical solution of the unsteady viscous flow outside of an expanding or contracting cylinder has been reported by Fang et al. [11].

The unsteady nature of the fluid flow is very important from a practical point of view. Some unsteady effects arise due to non-uniformities in the surrounding fluid. Other effects arise due to the self-induction of the body. In fact, there are some devices are designed to execute time-dependent motion in order to perform desired functions [12]. The understanding of unsteady flow and hence applying such knowledge to new design techniques enable scientists and engineers to make important improvements in reliability and costs of several fluid dynamics devices.

The problem introduced in this work involves such concept of unsteadiness. In fact, we investigate the case of unsteady viscous flow over a stretching horizontal cylinder with variable radius where the thermal radiation is considered. The understanding of unsteady flow and hence applying such knowledge to new design techniques enable scientists and engineers to make important improvements in reliability and costs of several fluid dynamics devices. Mathematica is used to solve the problem numerically. The obtained results show how the fluid velocity and temperature are affected by the variation of the parameters included in the study such as the radiation parameter, the unsteadiness parameter, and the suction/injection parameter.

\section{Mathematical formulation of the problem}

Consider an unsteady axisymmetric boundary layer flow of an incompressible viscous fluid along a horizontal cylinder which is considered to be continuously stretching. The cylinder is contracting or expanding according to the relation $a(t)=a_{0} \sqrt{1-\beta t}$, where $a(t)$ is the radius of the cylinder at any time $t, a_{0}$ is the initial value of the cylinder radius, and $\beta$ is a constant which indicates to contraction $(\beta>0)$ or expansion $(\beta<0)$.

The stretching time-dependent velocity of the surface of the cylinder is assumed to be $U_{w}(x, t)=\frac{4 v U_{0} x}{a^{2}(t)}$, and the fluid is assumed to move along the axial direction $x$ while the radial coordinate $r$ is perpendicular to the axis of the cylinder. The temperature of the cylinder surface is assumed to be time dependent in the form $T_{w}(x, t)=T_{\infty}$ $+\frac{a_{0} T_{0} x}{a(t)}$. Figure 1 shows the considered model. 


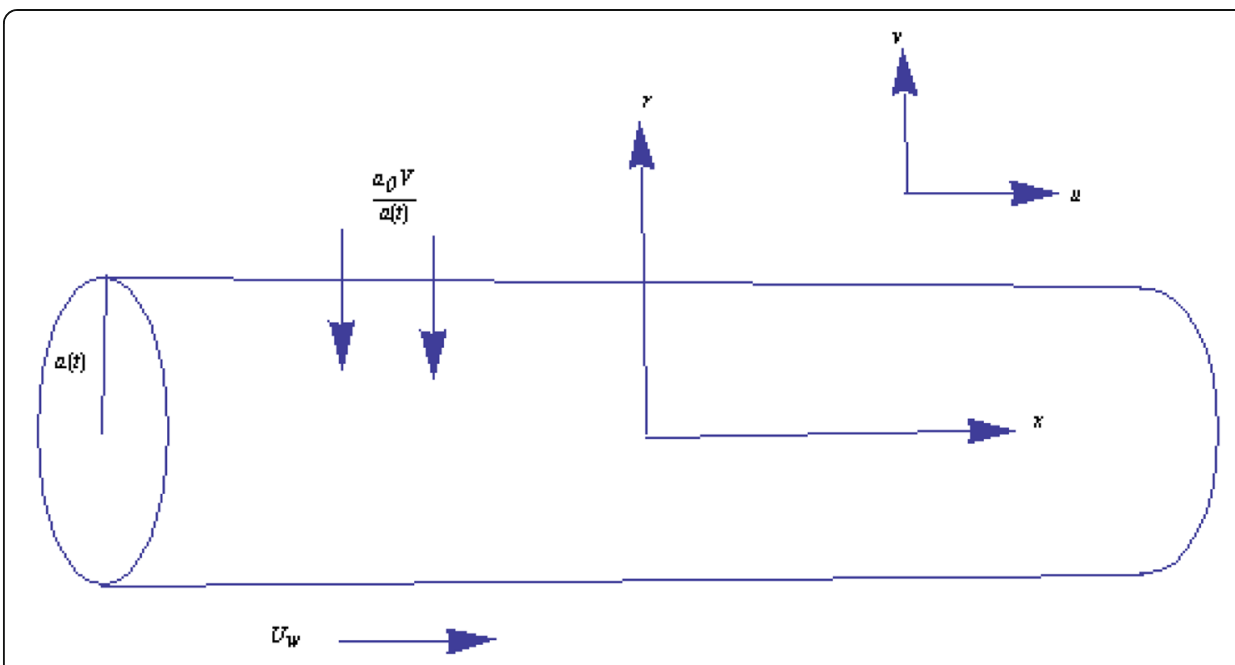

Fig. 1 The physical model

The governing equations $[13,14]$ are:

$$
\begin{aligned}
& \frac{\partial}{\partial x}(r u)+\frac{\partial}{\partial r}(r v)=0 \\
& \frac{\partial u}{\partial t}+u \frac{\partial u}{\partial x}+v \frac{\partial u}{\partial r}=\frac{v}{r} \frac{\partial}{\partial r}\left(r \frac{\partial u}{\partial r}\right) \\
& \frac{\partial T}{\partial t}+u \frac{\partial T}{\partial x}+v \frac{\partial T}{\partial r}=\frac{\alpha}{r} \frac{\partial}{\partial r}\left(r \frac{\partial u}{\partial r}\right)-\frac{\alpha}{\kappa} \frac{1}{r} \frac{\partial}{\partial r}\left(r q_{r}\right)
\end{aligned}
$$

the boundary conditions are:

$$
\begin{gathered}
u=U_{w}(x, t), v=\frac{a_{0} V}{a(t)}, T=T_{w}(x, t), \text { at } r=a(t) \\
u=0, T=T_{\infty}, \quad \text { as } r \rightarrow \infty
\end{gathered}
$$

where $u$ and $v$ are the components of the fluid velocity along $x$ axis and $r$ axis respectively. $v$ is the fluid kinematic viscosity, $\alpha$ is the fluid thermal diffusivity, $\kappa$ is the thermal conductivity, $V$ is the constant of suction $(V<0)$ or injection $(V>0)$, and $q_{r}$ $=-\frac{4 \sigma}{3} \alpha^{*} \frac{\partial T^{4}}{\partial r}$ is the radiation heat flux such that $\sigma$ and $\alpha^{*}$ are the Stefan-Boltzman constant and the mean absorption coefficient, respectively.

The temperature differences within the flow are assumed to be sufficiently small such that $T^{4}$ is expressed as a linear function of $T$; hence, the Taylor expansion of $T^{4}$ about $T_{\infty}$, neglecting higher order terms, is given by

Table 1 Comparison of $-f^{\prime \prime}(0)$ for various values of $A$ and $f_{0}$ given that $\operatorname{Pr}=0.7, U_{0}=-1, N_{R}=0$

\begin{tabular}{lllllll}
\hline$A$ & $f_{0}$ & Ref. [13] OHAM & Ref. [13] Numerical & Ref. [14] OHAM & Ref. [14] Numerical & Present Results \\
\hline-1 & -1 & 1.0000007 & 0.9999999 & 1.0000000 & 1.0000002 & 1.0000000 \\
-1 & -2 & 2.5632048 & 2.5632048 & 2.5632043 & 2.5632048 & 2.5632049 \\
-2 & -1 & 2.6012207 & 2.6012207 & 2.6012207 & 2.6012207 & 2.6012207 \\
-2 & -2 & 3.7150911 & 3.7150910 & 3.7150910 & 3.7150910 & 3.7150910 \\
\hline
\end{tabular}




$$
T^{4}=4 T_{\infty}^{3} T-3 T_{\infty}^{4}
$$

Considering the similarity transformations

$$
\eta=\frac{r^{2}}{a^{2}(t)}-1, u=\frac{U_{w}}{U_{0}} f^{\prime}(\eta), v=-\frac{2 v}{r} f(\eta), \theta=\frac{T-T_{\infty}}{T_{w}-T_{\infty}}
$$

along with Eq. (6), the system of partial differential Eqs. (1)-(3) with the boundary conditions (4)-(5) is transformed into the following system of ordinary differential equation

$$
\begin{aligned}
& (1+\eta) f^{\prime \prime \prime}+f^{\prime \prime}+f f^{\prime \prime}-f \prime^{2}-A\left[(1+\eta) f^{\prime \prime}+f^{\prime}\right]=0 \\
& \frac{1}{P r}\left[\left(1+N_{R}\right)\left((1+\eta) \theta^{\prime \prime}+\theta^{\prime}\right)\right]+f \theta^{\prime}-f^{\prime} \theta-A\left[(1+\eta) \theta^{\prime}+\frac{\theta}{2}\right]=0
\end{aligned}
$$

subject to the boundary conditions:

$$
\begin{aligned}
& f(0)=-f_{0}, f^{\prime}(0)=U_{0}, \theta(0)=1 \\
& f^{\prime}(\infty) \rightarrow 0, \theta(\infty) \rightarrow 0
\end{aligned}
$$

while primes denote differentiation with respect to $\eta, A=\frac{\beta a_{0}^{2}}{4 v}$ is the unsteadiness parameter. Where the negative values of $A$ correspond to contraction and the positive values of $A$ correspond to expansion, $\operatorname{Pr}=\frac{v}{\alpha}$ is the Prandtl number, $f_{0}=\frac{a_{0} V}{2 v}$ is the suction $\left(f_{0}<0\right)$ or injection $\left(f_{0}>0\right)$ parameter, and $N_{R}=\frac{16 \sigma T_{\infty}^{3}}{3 \kappa \alpha^{*}}$ is the thermal radiation parameter.

Two important physical quantities of interest are the skin friction, $C_{f}$, and the local Nusselt number, $N u_{x}$, which are defined as:

$$
C_{f}=\frac{2 \tau_{w}}{\rho U_{w}^{2}}, N u_{x}=\frac{x\left(q_{w}+q_{r}\right)}{\kappa\left(T_{w}-T_{\infty}\right)}
$$

where $\tau_{w}=\mu\left(\frac{\partial u}{\partial r}\right)_{r=a(t)}$ is the cylinder surface sheer stress and $q_{w}=-\kappa\left(\frac{\partial T}{\partial r}\right)_{r=a(t)}$ is the cylinder surface heat flux. Using the dimensionless similarity transformations (7), we get:

$$
\frac{U_{0}}{2} C_{f} \sqrt{U_{0} R e_{x}}=f^{\prime \prime}(0), N u_{x} \sqrt{U_{0} / R e_{x}}=-\left(1+N_{R}\right) \theta^{\prime}(0)
$$

where $R e_{x}=\frac{x U_{w}}{v}$ is the Reynolds number.

\section{Method of solutions}

Equations (8)-(9) subject to the boundary conditions (10)-(11) are transformed into the following system of first-order differential equations:

$$
\begin{aligned}
& y \prime_{1}=y_{2} \\
& y_{2}=y_{3} \\
& y \prime_{3}=\frac{1}{(1+\eta)}\left[A\left((1+\eta) y_{3}+y_{2}\right)+y_{2}^{2}-y_{3}-y_{1} y_{3}\right] \\
& y \prime_{4}=y_{5}
\end{aligned}
$$


Table 2 Values of $-f^{\prime \prime}(0)$ and $-\theta^{\prime}(0)$ for various values of $A, \operatorname{Pr}, f_{0}$, and $N_{R}$ for $U_{0}=1$

\begin{tabular}{llllll}
\hline$A$ & $P r$ & $f_{0}$ & $N_{R}$ & $-f^{\prime \prime}(0)$ & $-\theta^{\prime}(0)$ \\
\hline-2 & & & 3.3170862 & 1.8884144 \\
-1 & 0.7 & -1 & 0.5 & 2.4515742 & 1.4338464 \\
1 & & & 1.4434620 & 0.8077094 \\
-1 & 0.7 & -1 & 0.5 & 2.4515742 & 1.4338464 \\
& 1 & & & 2.4515742 & 1.9023636 \\
& 10 & -2 & & 2.4515742 & 14.3735363 \\
-1 & & -1 & 0.5 & 3.3307422 & 1.7883330 \\
& 0.7 & 1 & & 2.4515742 & 1.4338464 \\
& & & 0.5 & 2.0150457 & 0.1622559 \\
-1 & & & 0.7 & 2.4515742 & 1.4338464 \\
& & & 1 & 2.4515742 & 1.3019903 \\
& & & & 2.4515742 & 1.1515467 \\
\hline
\end{tabular}

$$
y_{5}^{\prime}=\frac{P r}{\left(1+N_{R}\right)(1+\eta)}\left[y_{2} y_{4}+A\left(\frac{y_{4}}{2}+(1+\eta) y_{5}\right)-y_{1} y_{5}\right]-\frac{y_{5}}{(1+\eta)}
$$

where $y_{1}=f, y_{2}=\dot{f}, y_{3}=\dot{f}^{\prime}, y_{4}=\theta, y_{5}=\theta^{\prime}$

and the initial conditions are then:

$$
y_{1}(0)=-f_{0}, y_{2}(0)=U_{0}, y_{4}(0)=1, y_{3}(0)=m, y_{5}(0)=n
$$

numerical values are given to $U_{0}$ and $f_{0} . m$ and $n$ are priori unknown to be determined as part of the solution. Mathematica is used to define the function $F[m, n]=$ : NDSolve[System(14) - (19)]. The values of $m$ and $n$ are found upon solving the equations $y_{2}\left(\eta_{\max }\right)=0, y_{4}\left(\eta_{\max }\right)=0$. A suitable value of $\eta$ is taken and then increased to reach $\eta_{\max }$ such that the difference between successive values of $m$ and those of $n$ is less than $10^{-7}$. The problem now is an initial value problem which in turn is solved using the Mathematica function NDSolve.

\section{Special cases}

The accuracy of the numerical method is checked out by comparing results in some special cases with previously published results in the literature as shown in Table 1.

From the table, one can find a comparison of the obtained values of $-f^{\prime}(0)$ with previously published results in the literature. The comparison is made for various values of $A$ and $f_{0}$ given $\operatorname{Pr}=0.7, U_{0}=-1, N_{R}=0$. The obtained results show good amendment which gives rise to the validation of the used method.

\section{Results and discussions}

This section is devoted to the analysis of the behavior of the parameters included in the problem on the fluid velocity $\dot{f}(\eta)$, the fluid temperature $\theta(\eta)$, modified skin friction $-\dot{f}$ '(0) and the modified Nusselt number $-\theta$ ' $(0)$.

Table 2 shows the effects of $A, \operatorname{Pr}, f_{0}$, and $N_{R}$ on both of $-f^{\prime}(0)$ and $-\theta^{\prime}(0)$ for $U_{0}=1$. It can be observed that the values of $-f^{\prime}(0)$ are positive for all values of the considered parameters. The physical interpretation of this behavior is that the cylinder surface 


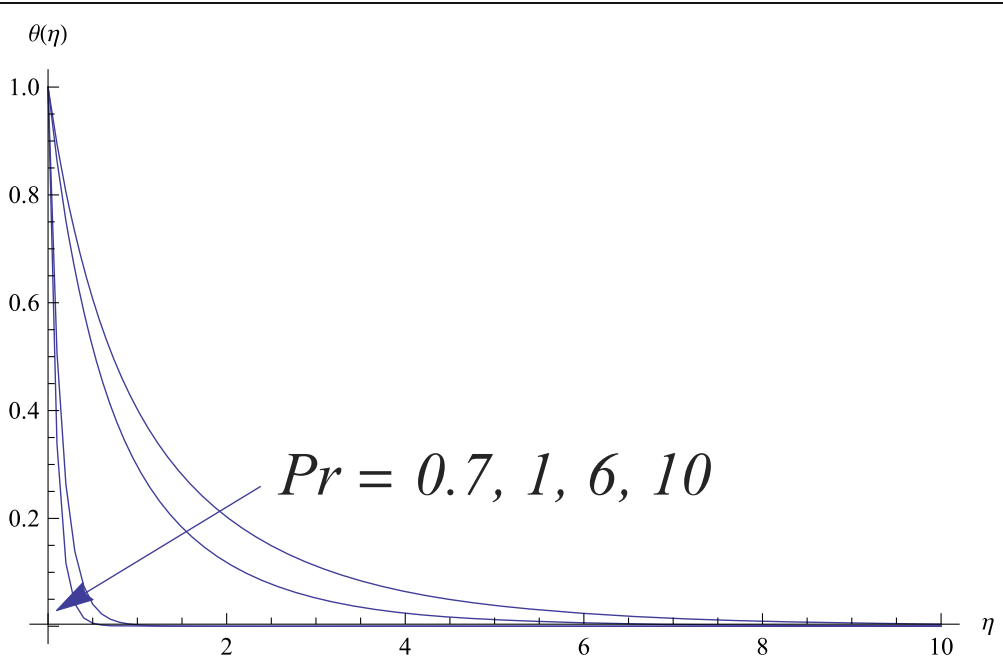

Fig. 2 Variation of fluid temperature with $\operatorname{Pr}$, for $A=-1, N_{R}=1, U_{0}=1, f_{0}=-1$

exerts a drag force on the fluid which is understood in view of the role of the stretching cylinder to induce the flow when $U_{0}=1$.

\section{Prandtl number}

As the Prandtl number increases the thermal conductivity of the fluid decreases so the surface heat flux (Nusselt number) increases which is consistent with the results shown in Table 2. The increasing of the Nussselt number results in decreasing the temperature of the fluid as shown in Fig. 2. At a distance far enough from the surface of the cylinder, the fluid temperature becomes the same as that of the ambient fluid.

\section{Thermal radiation}

The effect of thermal radiation on the fluid temperature is easily recognized from Table 2 and Fig. 3. Increasing $N_{R}$ leads to the decrease of the surface heat flux (Nusselt

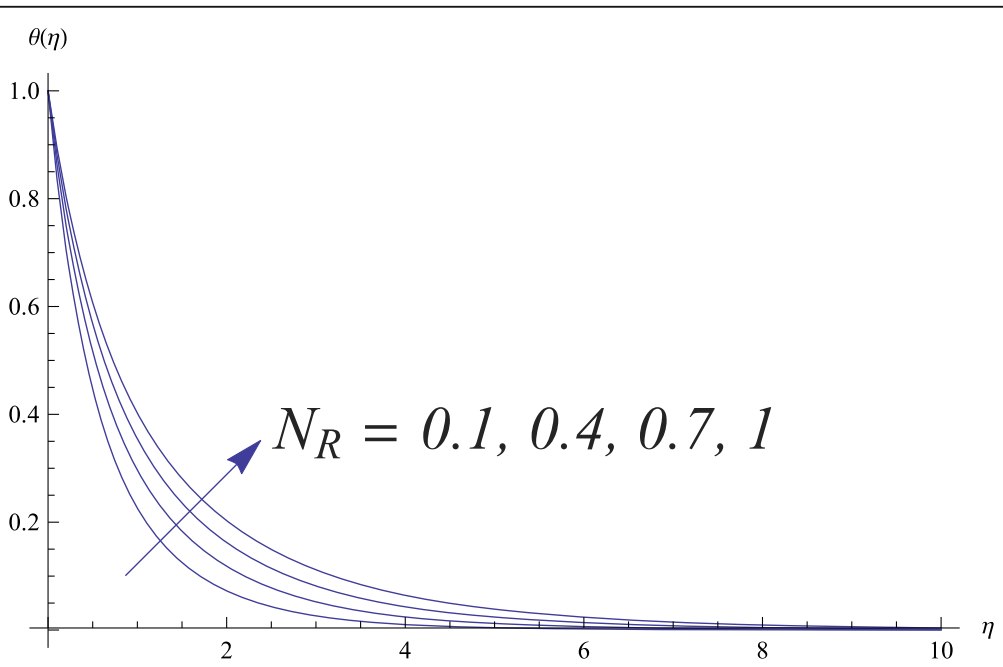

Fig. 3 Variation of fluid temperature with $N_{R^{\prime}}$ for $A=-1, \operatorname{Pr}=0.7, U_{0}=1, f_{0}=-1$ 


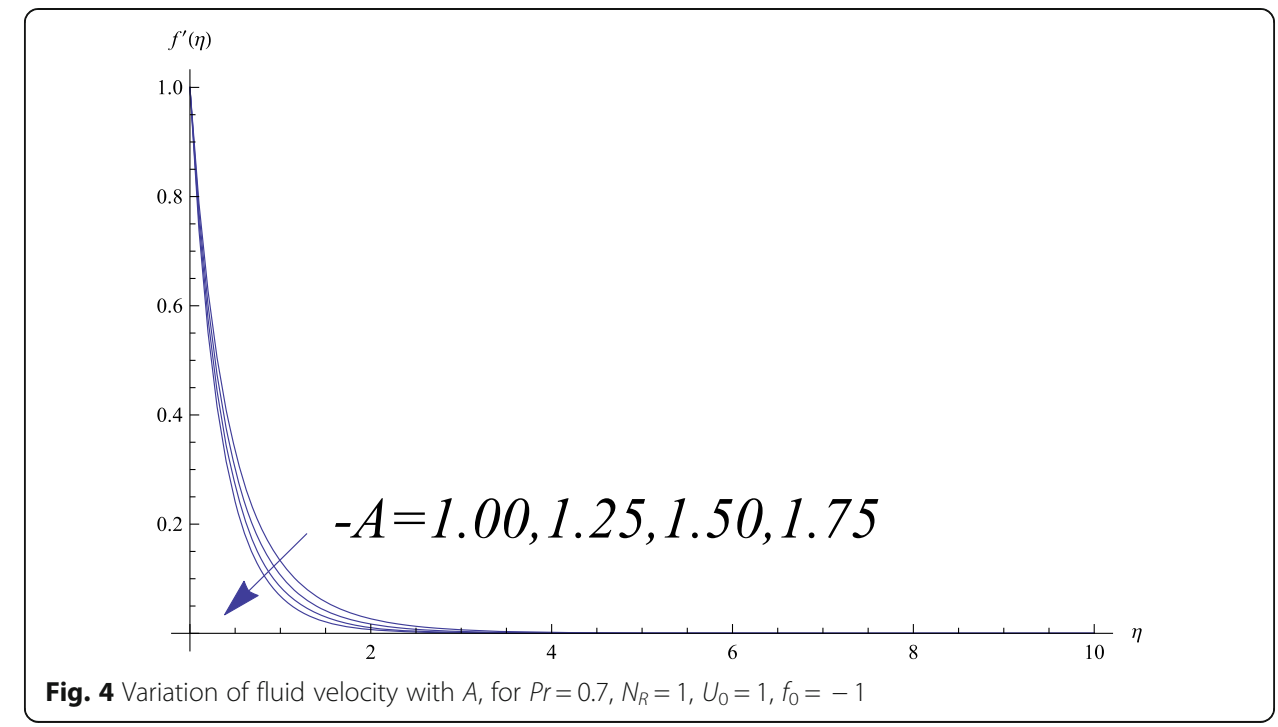

number) and hence the fluid temperature increases. The physical interpretation of this behavior is that as the value of $N_{R}$ increases the Rosseland radiation absorptivity $\alpha^{*}$ decreases and hence the radiative heat flux $\frac{\partial q_{r}}{\partial r}=-\frac{4 \sigma}{3 \alpha^{*}} \frac{\partial T^{4}}{\partial r}$ increases and consecutively increases the rate of radiative heat transferred to the fluid and hence the fluid temperature elevates.

The equations governing the fluid velocity does not include $N_{R}$ so there is no effect on $N_{R}$ on the skin friction or the fluid velocity as recognized from Table 2.

\section{Expandable unsteadiness parameter}

For negative values of $A$, as $A$ decreases, the cylinder expands. Such expansion forces the cylinder surface to be closer to the fluid. So, the frictional forces between the fluid particles and the surface of the cylinder elevate which results in increasing the skin friction as observed in Table 2 and decreasing the fluid velocity as figured out in Fig. 4. It

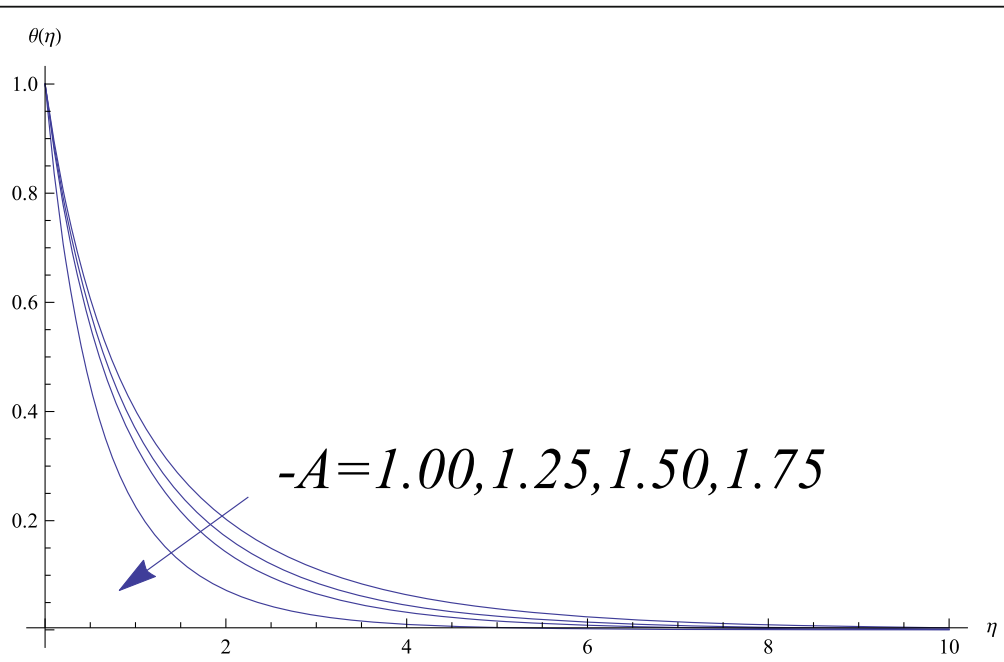

Fig. 5 Variation of fluid temperature with $A$, for $\operatorname{Pr}=0.7, N_{R}=1, U_{0}=1, f_{0}=-1$ 


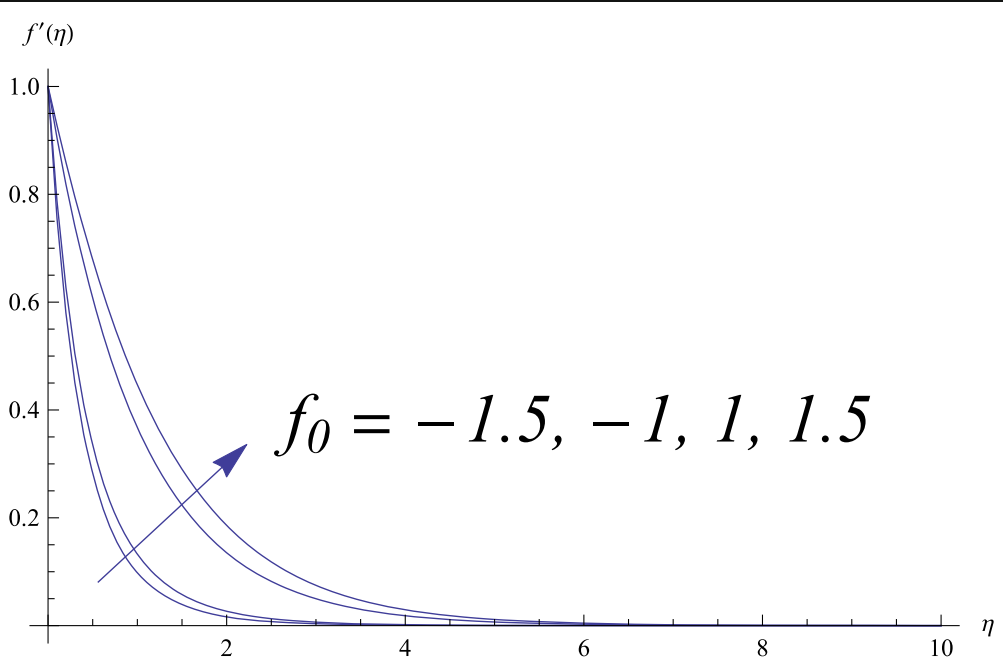

Fig. 6 Variation of fluid velocity with $f_{0}$, for $\operatorname{Pr}=0.7, N_{R}=1, U_{0}=1, A=-1$

can also be noted from Table 2 that the decreasing of $A$ increases the Nusselt number so the rate of heat transfer elevates which in turn decreases the fluid temperatures as depicted in Fig. 5.

\section{Suction/injection velocity}

The value of $f_{0}$, the suction/injection parameter, plays an important role in controlling the friction between the fluid and the surface of the cylinder which in turn affects the heat transfer rate at the cylinder surface. Suction causes the streamlines gets closer to the cylinder surface so the skin friction increases by increasing the value of the suction parameter as shown in Table 2. Consequently, the friction between the fluid layers increases which enforce the fluid to slow down and the fluid velocity gradient decreases as shown in Fig. 6. From Table 2, we can also find that the values of $-f^{\prime}(0)$ are bigger for suction in comparison to injection. Such observations imply the conclusion that

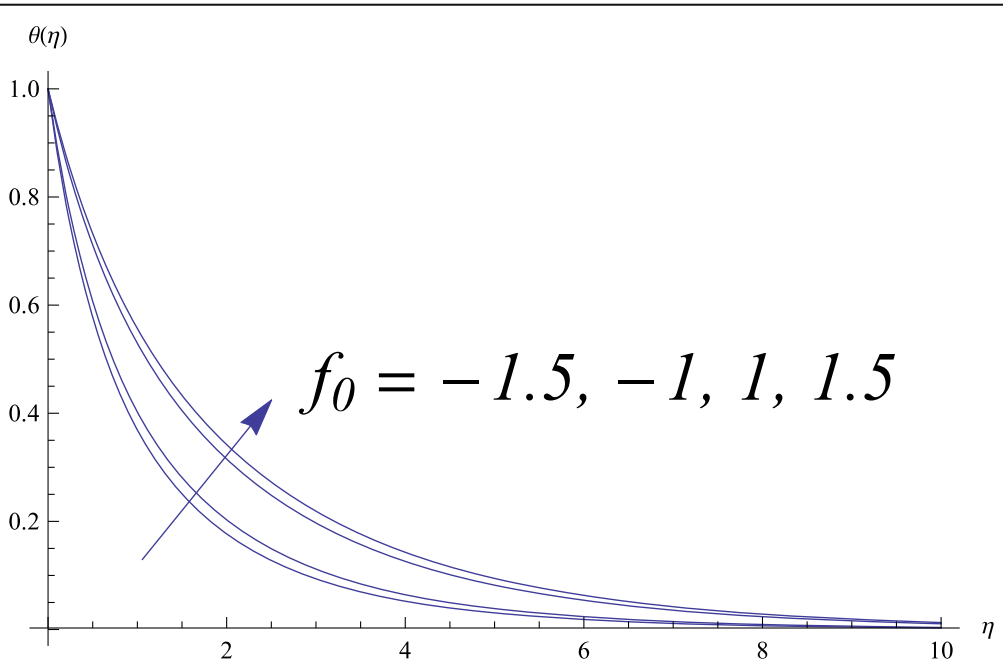

Fig. 7 Variation of fluid temperature with $f_{0}$ for $\operatorname{Pr}=0.7, N_{R}=1, U_{0}=1, A=-1$ 
introducing the injection may help to reduce the friction at the surface of the cylinder. However, the velocity gradient vanishes at some distance large enough from the surface of the cylinder.

The value of Nusselt number in the injection case is smaller than that of suction as recognized from Table 2, and consequently, the fluid temperature is higher as depicted in Fig. 7. This behavior can be justified physically as follows: the lateral mass flux in case of injection enhances the thermal conductivity of the fluid, and hence, the amount of temperature that poured from the cylinder surface to the fluid increases which results in decreasing the Nusselt number. The inverse behavior takes place in case of suction.

\section{Conclusions}

The problem of radiative fluid flow and heat transfer of a fluid along an expandable-stretching horizontal cylinder has been studied. The study results are the following:

- With the decrease of the value of the contraction parameter, the skin friction increases while both of the fluid velocity and temperature decrease.

- Increasing the Prandtl number leads to a decrease of the Nusselt number and the fluid temperature as well.

- The fluid velocity decreases as suction increases while the increase of injection leads to increasing the fluid velocity

- The increase of injection enhances the fluid temperature while the inverse behavior of takes place in the case of suction

- The surface flux (Nusselt number) and consequently the fluid temperature increase as the thermal radiation parameter increases.

- Introducing the injection may help to reduce the friction at the surface of the cylinder

\section{Nomenclature}

$t$ : Time $[\mathrm{s}]$; $a(t)$ : Radius of the cylinder $[\mathrm{m}] ; U_{w}$ : Stretching time-dependent velocity $\left[\mathrm{m} \mathrm{s}^{-1}\right]$; $x$ : Axial direction coordinate $[\mathrm{m}] ;$ : Perpendicular to the axis coordinate $[\mathrm{m}]$; $u$ : Velocity component in the $x$-direction $\left[\mathrm{m} \mathrm{s}^{-1}\right]$; $v$ : Velocity component in the $r$-direction $\left[\mathrm{m} \mathrm{s}^{-1}\right] ; T_{w}$ : Temperature of the cylinder surface $[\mathrm{K}] ; \mathrm{V}$ : Constant of suction [-]; A: Unsteadiness

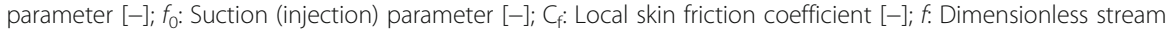
function [-]; $N_{\mathrm{R}}$ : Radiation parameter [-]; $N u_{x}$ : The local Nusselt number coefficient [-]; Pr: Prandtl number [-]; Re $_{x}$ : Reynolds number $[-] ; q_{r}$ : Cylinder surface heat flux $\left[\mathrm{kg} \mathrm{s}^{-3}\right]$

\section{Greek symbols}

$a$ The thermal diffusivity $\left[\mathrm{m}^{2} \mathrm{~s}^{-1}\right]$

$a^{*}$ Mean absorption coefficient [-]

$\beta$ Contraction (expansion) constant $\left[\mathrm{s}^{-1}\right]$

$\eta$ The dimensionless similarity variable $[-]$

$u$ Kinematic viscocity $\left[\mathrm{m}^{2} \mathrm{~s}^{-1}\right]$

$\mu$ Dynamic viscocity $\left[\mathrm{m}^{2} \mathrm{~s}^{-1}\right]$

$K$ Thermal conductivity of the fluid $\left[\mathrm{kg} \mathrm{m} \mathrm{s}^{-3} \mathrm{~K}^{-1}\right]$

\section{Acknowledgements}

The author want to thank the reviewers for their valuable comments which enabled the author to improve the manuscript.

Funding

This work has been accomplished in the faculty of science and arts- Khulais, university of Jeddah where I work as associate professor. 
Authors' contributions

The author read and approved the final manuscript.

\section{Competing interests}

The author declares that he has no competing interests.

\section{Publisher's Note}

Springer Nature remains neutral with regard to jurisdictional claims in published maps and institutional affiliations.

Received: 18 June 2018 Accepted: 9 September 2018

Published online: 04 April 2019

\section{References}

1. Uchida, S., Aoki, H.: Unsteady flows in a semi-infinite contracting or expanding pipe. J. Fluid Mech. 82(2), 371-387 (1977)

2. Skalak, F.M., Wang, C.Y.: On the unsteady squeezing of viscous fluid from a tube. J. Aust. Math. Soc. B. 21, 65-74 (1979)

3. Wang, C.Y.: Fluid flow due to a stretching cylinder. Phys. Fluids. 31, 466-468 (1988)

4. Elbashbeshy, E.M.A., Emam, T.G., Elazab, M.S., Abdelgaber, K.M.: Effect of magnetic field on flow and heat transfer over a stretching horizontal cylinder in the presence of a heat source/sink with suction/injection. J. Appl. Mech. Eng. 1(1), 1-5 (2012)

5. Hayat, T., Waqas, M., Shehzad, S.A., Alsaedi, A.: Mixed convection flow of viscoelastic nanofluid by a cylinder with variable thermal conductivity and heat source/sink. Int. J. Numer. Methods Heat Fluid Flow. 26(1), 214-234 (2016)

6. Ishak, A., Nazar, R., Pop, I.: Magnetohydrodynamic (MHD) flow and heat transfer due to a stretching cylinder. Energ. Convers. Manage. 49(11), 3265-3269 (2008)

7. Mabood, F., Shateyi, S., Khan, W.A.: Effect of thermal radiation on Casson flow heat and mass transfer around a circular cylinder in a porus medium. Eur. Phys. J. Plus. 130, 188 (2015)

8. Zaimi, K., Ishak, A., Pop, I.: Unsteady flow due to a contracting cylinder in a nanofluid using Buongiorno's model. Int. J. Heat Mass Transf. 68, 509-513 (2014)

9. El-Bashbeshy, E.M.A., Emam, T.G., Abdelwahed, M.S.: The effect of thermal radiation, heat generation, and suction/ injection on the mechanical properties of unsteady continuous moving cylinder in a nanofluid. Therm. Sci. 19(5), 15911601 (2015)

10. Fang, T., Zhang, J., Zhong, Y., Tao, H.: Unsteady viscous flow over an expanding stretching cylinder. Chin. Phys. Lett. 28, 124707 (2011)

11. Fang, T., Zhang, J., Zhong, Y.: Note on unsteady viscous flow on the outside of an expanding or contracting cylinder Commun. Nonlinear Sc. Num. Simul. 17, 3124-3128 (2012)

12. McCrosky, W.J.: Some current research in unsteady fluid dynamics - the 1976 Freeman scholarship lecture. ASME J. Fluid Eng. 99, 8-39 (1977)

13. Marinca, V., Ene, R.D.: Dual approximate solutions of the unsteady viscous flow over a shrinking cylinder with optimal homotopy asymptotic method. Adv. Math. Phys. 2014, 417643, 11 (2014)

14. E.M.A. Elbashbeshy, T.G. Emam, and K.M. Abdelgaber, Semi-analytic and numerical solutions of unsteady flow and heat transfer of a fluid over an expandable-stretching horizontal cylinder in the presence of suction/injection, preprint. (2014)

\section{Submit your manuscript to a SpringerOpen ${ }^{\circ}$ journal and benefit from:}

- Convenient online submission

- Rigorous peer review

- Open access: articles freely available online

- High visibility within the field

- Retaining the copyright to your article

Submit your next manuscript at $\boldsymbol{\nabla}$ springeropen.com 